\section{Case Reports in Orthopedic Research}

\title{
Successful Early Non-Operative Management of Congenital Knee Dislocation: Demonstration via a Case Report
}

\author{
Punit Tiwari $^{a}$ Harmeet Kaur $^{\mathrm{b}}$ Sergey S. Leonchuk ${ }^{c}$ \\ aDepartment of Orthopaedics, Maharishi Markandeshwar Medical College and Hospital, \\ Kumarhatti, Solan, India; bepartment of Radiodiagnosis, All India Institute of Medical \\ Sciences, Bathinda, India; 'Head of 6th Orthopedic Department, National Ilizarov Medical \\ Research Center for Traumatology and Orthopedics of Ministry of Healthcare, Kurgan City, \\ Russia
}

\section{Keywords}

Congenital knee dislocation · Treatment · Reverse knee joint · Serial casting · Classification

\begin{abstract}
Congenital knee dislocation (CKD) is a deformity in which unnatural hyperextension is possible at the knee much beyond the normal $0^{\circ}$ full extension while the flexion is severely restricted. Incidence of this rare congenital malformation is $1 / 100,000$ live births. We present a clinical case of unilateral CKD in a 2-week-old female child and our approach of treatment using serial casting. We have been able to demonstrate that if initiated early, within first few weeks of life, the congenital deformity of knee can be managed conservatively without any surgical intervention even in higher grades.
\end{abstract}

\section{Introduction}

Congenital knee dislocation (CKD) is a deformity in which unnatural hyperextension is possible at the knee much beyond the normal $0^{\circ}$ full extension while the flexion is severely restricted. It may either be idiopathic or a part of any syndrome (Larsen's syndrome, arthrogryposis multiplex congenita, or myelomeningocele) [1-3]. As the incidence of CDK is very low (approximately $1 / 100,000$ live births $[4,5]$ which is about $1 \%$ of the incidence of congenital hip dislocation [6]), so many paediatricians and orthopaedicians often have not seen even a single such case. The deformity may be unilateral or bilateral and mostly affect 
Case Reports

in Orthopedic

Research

\begin{tabular}{l|l}
\hline Case Rep Orthop Res 2021:4:138-144 \\
\hline DOI: 10.1159/000516134 & $\begin{array}{l}\text { @ 2021 The Author(s). Published by S. Karger AG, Basel } \\
\text { www.karger.com/cio }\end{array}$ \\
\hline
\end{tabular}

Tiwari et al.: Successful Early Non-Operative Management of Congenital Knee Dislocation

girls but some literature reports equal distribution [7-9]. Due to the lack of knowledge and experience regarding the management, the health-care providers are not able to explain and guide the parents regarding the prognosis and management of this congenital deformity. The purpose of this study is to share our experience of conservative management of a case of CKD at birth and the problems encountered during the process.

\section{Case Presentation}

A 2-week-old female child was brought to the outdoor patient department for consultation regarding a "reverse knee joint" in the left lower limb since birth (shown in Fig. 1a-c). The child was born via normal vaginal delivery by a traditional birth attendant. The parents had earlier taken the child to nearby doctors who could diagnose the condition but could not offer a treatment plan. Ultimately, the child was referred to our institute.

Deformity and knee hyperextension were so severe that the infant's resting position was with the foot adjacent to the head. Unfortunately, parents did not consent for X-rays at that early age; instead, knee ultrasonography was performed (shown in Fig. 2). Developmental dysplasia of the hip was ruled out with the Barlow and Ortolani test, corroborated with hip ultrasonography. The dislocation could be gently reduced by posteroanterior "piston" but unstable and recurred once the pressure on the condyles was relaxed.

As the passive flexion was not possible at all at the initial visit, that is, $<30^{\circ}$, so it fell in group 3 of Abdelaziz and Samir [1] grading of CDK and so V-Y quadricepsplasty was recommended. In the presented case, the patient had deep anterior skin grooves and fell in type II of Mehrafshan et al. [10] reduction and stability criteria. However, the parents did not consent for the operative intervention.

So, the serial weekly casting was started and $>90^{\circ}$ passive as well as active flexion could be achieved at the end of the sixth cast. Then the cast was discontinued and a back splint has been given to the patient to be used at night-time only. The splint is removed during daytime or whenever the child is awake to encourage active flexion and extension at the knee. Parents of the child are quite satisfied with the results achieved (shown in Fig. 3).

\section{Complications}

After the second cast there was a development of a huge blister over and around the left knee in spite of taking all precautions, so the casting had to be discontinued for 2 weeks (shown in Fig. 4). The blister healed uneventfully and the corrective casting was resumed with extra padding.

We took the utmost care to be as gentle as possible and never be forceful while doing the manipulation. Each time we change the cast, our immobilization should be stable enough to hold the incremental correction we achieve. The role of adequate padding cannot be overemphasized in this technique. While the upper extent of our plaster cast was till groin, however, the lower extent has been just above the ankle joint which was kept free. This required extra care by parents to prevent the soiling of cast near the perineal region while maintaining toilet hygiene.

On the initial ultrasound examination, it was found that the epiphyseal ossification centre of the proximal end of the left tibia had not appeared and it was confirmed on subsequent $\mathrm{X}$-rays, though the ossification centre of the left distal femur and both distal right femur and right proximal tibia ossification centres were present. This finding is important because any

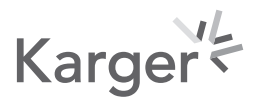




\section{Case Reports in Orthopedic Research}
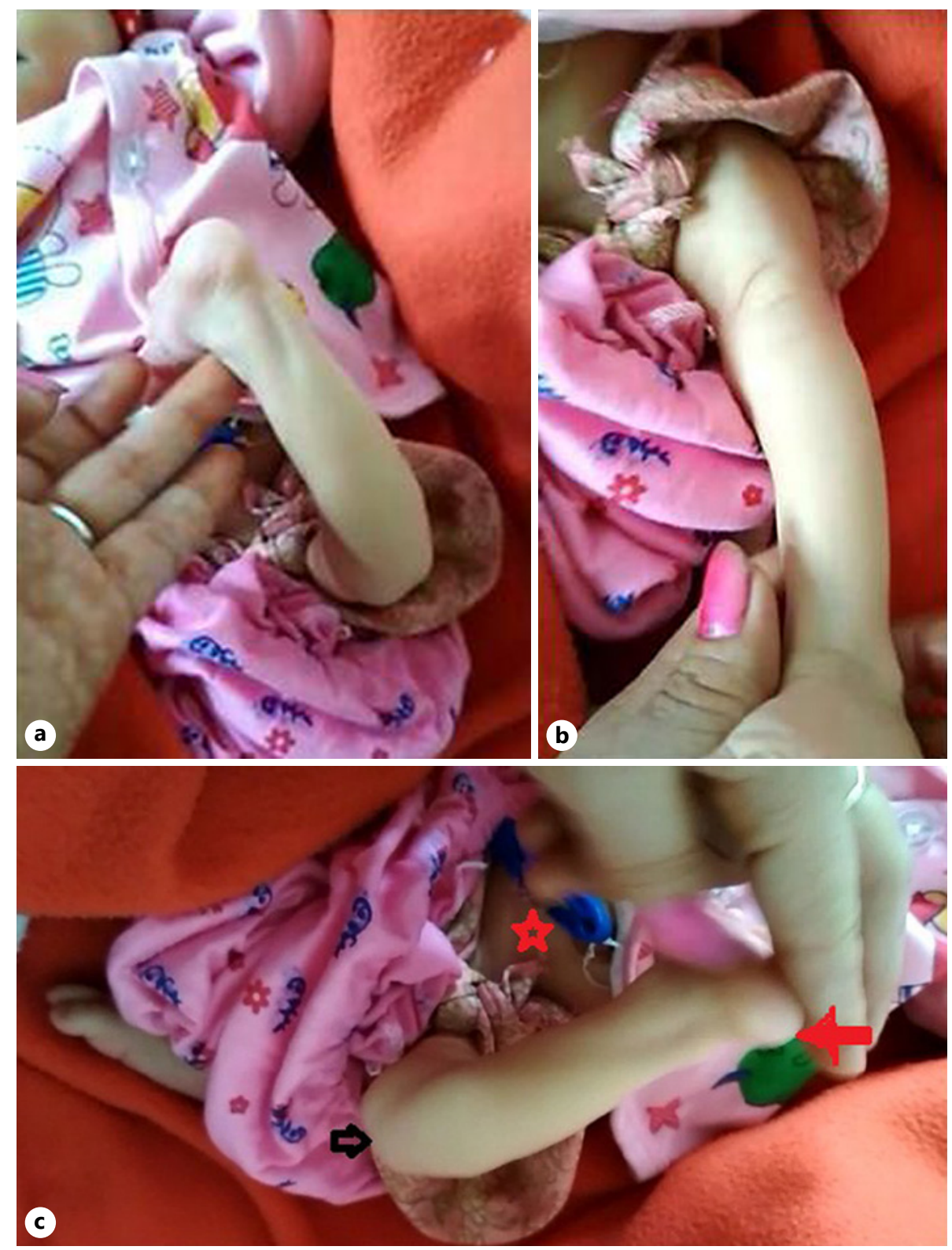

Fig. 1. a Spontaneous position of left lower limb in a 2-week-old female child. b Maximum possible passive flexion of left knee. $\mathbf{c}$ Femoral condyles prominent posteriorly because of anterior dislocation of tibia (black arrow), left heel (red arrow), and navel area (red star).

future growth disturbances in the left lower limb under treatment could be attributed to growth plate damage during the serial casting procedure. Parents have been made aware of it before initiating treatment and explained the possible consequences.

After the discontinuation of serial casting, when $>90^{\circ}$ flexion had been achieved, a check $\mathrm{X}$-ray was done which revealed periosteal elevation of long bones of the left lower limb which may be due to periosteal stripping/separation while recurvatum deformity correction (shown in Fig. 5a, b).

Though this periosteal separation is of no consequence, still it will be followed up. Patella in comparison to the other side is smaller and high riding, but no significant difference in the range of motion of both knees. 


\section{Case Reports in Orthopedic Research}

\begin{tabular}{l|l}
\hline Case Rep Orthop Res 2021:4:138-144 \\
\hline DOI: 10.1159/000516134 & $\begin{array}{l}\text { @ 2021 The Author(s). Published by S. Karger AG, Basel } \\
\text { www.karger.com/cio }\end{array}$ \\
\hline
\end{tabular}

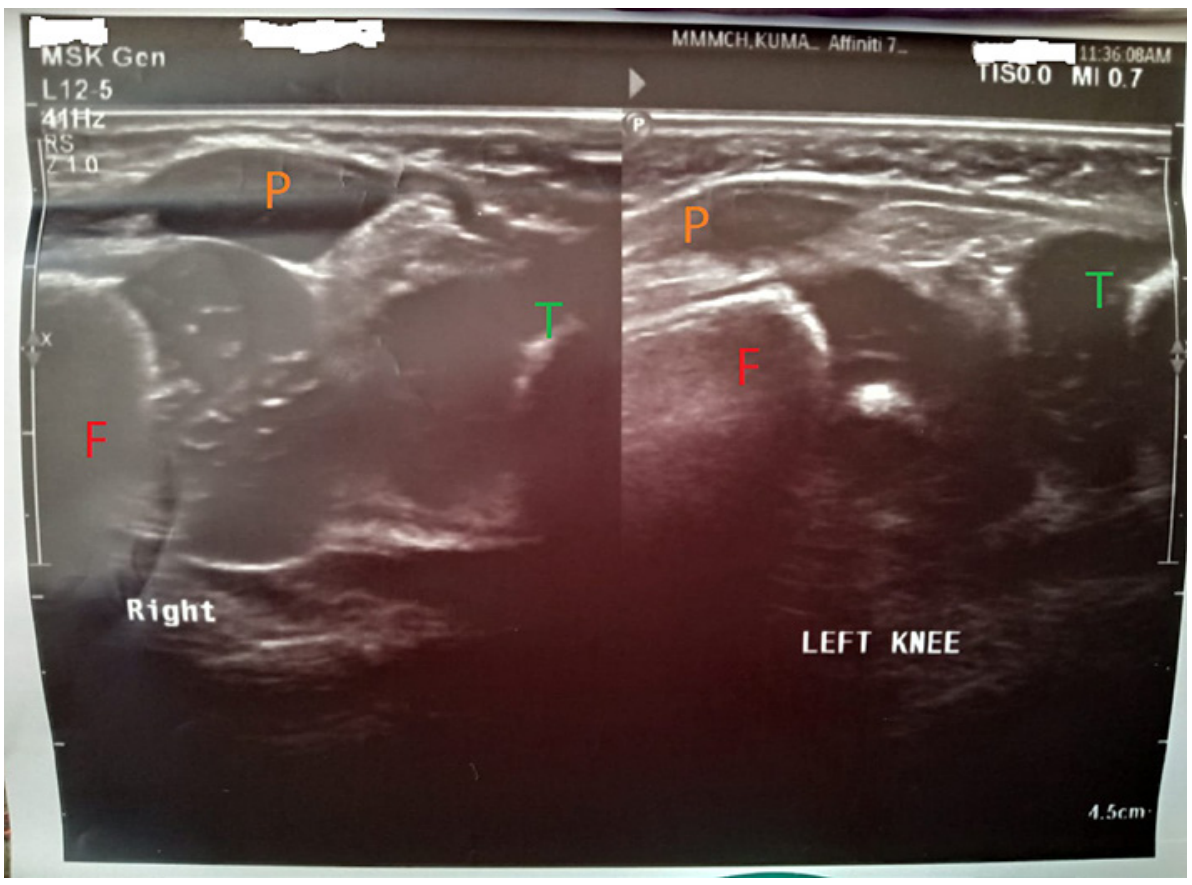

Fig. 2. Ultrasonography shows the smaller and high-riding patella, tight patellar tendon on the affected left side as compared to the normal right knee joint. F, femur; T, tibia; P, patella.

Fig. 3. Photograph of lower extremities (left knee after our treatment).

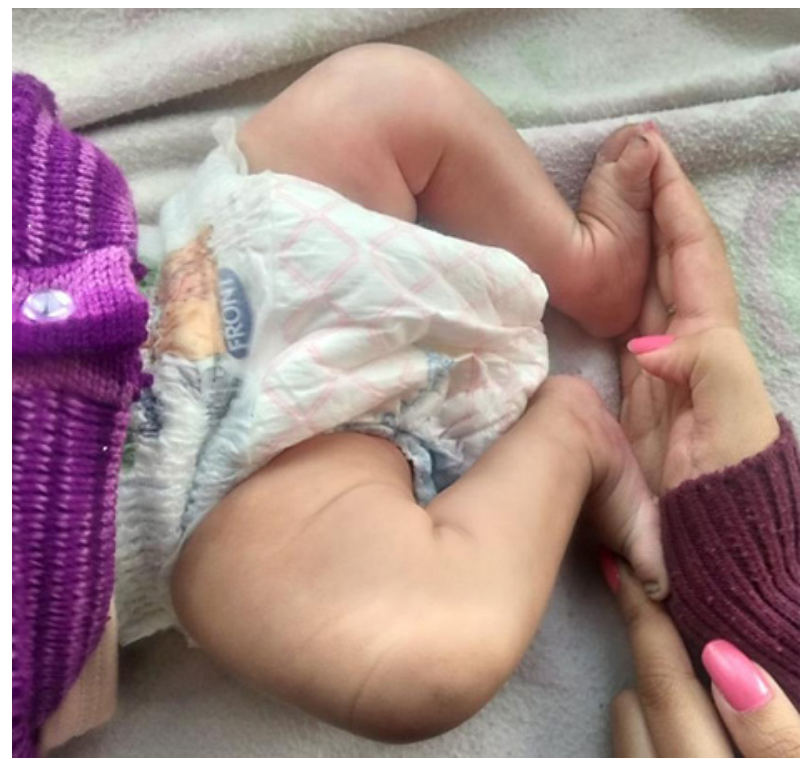

\section{Discussion}

Successful conservative management in cases of CDK can be easily done if it is diagnosed early after birth and timely treatment initiated. For this, awareness should be made in both the paediatric and orthopaedic community. CDK is presumed to develop in the second half of pregnancy, so the radiologist should be watchful of it in the prenatal ultrasound [11]. It may be a part of a syndrome so thorough examination is a must in such cases to rule out other associated anomalies. 


\section{Case Reports in Orthopedic Research}

\begin{tabular}{l|l}
\hline Case Rep Orthop Res 2021;4:138-144 \\
\hline DOI: 10.1159/000516134 & $\begin{array}{l}\text { C 2021 The Author(s). Published by S. Karger AG, Basel } \\
\text { www.karger.com/cio }\end{array}$ \\
\hline
\end{tabular}

Tiwari et al.: Successful Early Non-Operative Management of Congenital Knee Dislocation

Fig. 4. Large blister: complication during process of casting.
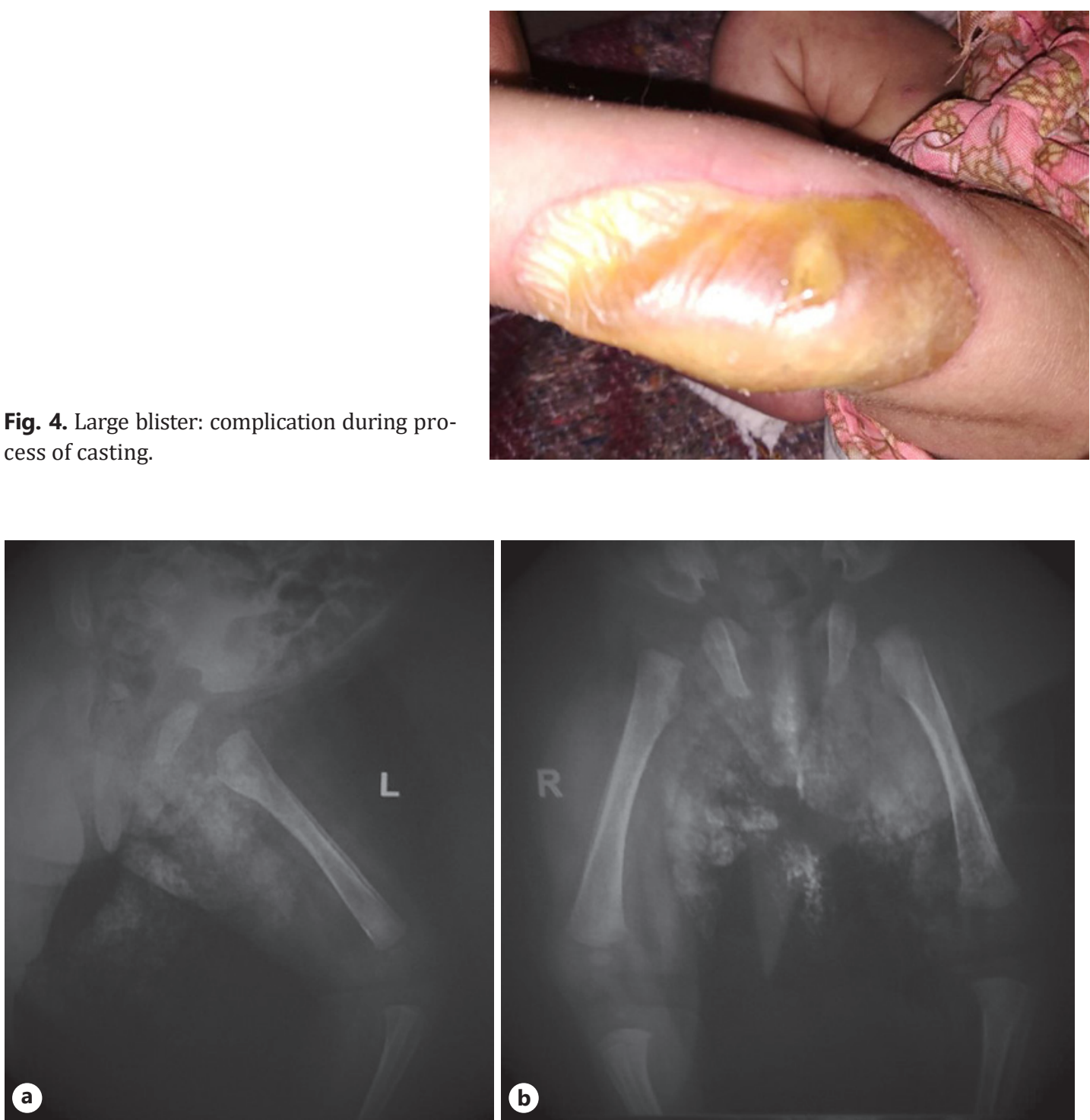

Fig. 5. a Lateral view X-rays of low extremities after treatment (periosteal elevation of long bones of left lower limb and left proximal tibia epiphysis is not visible). b AP-view X-rays of low extremities after treatment (periosteal elevation of long bones of left lower limb and left proximal tibia epiphysis is not visible).

Two well-known classifications, one by AbdelAziz and Samir [1] and another by Mehfershan et al. [10], are freely available in the literature to grade the severity and to guide the treatment plan. However, we found the latter to be less rigid and better in individual case assessment and treatment protocol. [12].

Radiological investigations before and after treatment have an important role to play especially with regard to growth plate and iatrogenic fractures. Both early and late complications of CDK like knee instability [5], cruciate ligament abnormality [13], and limb length discrepancies should be discussed with parents of the child well before starting treatment.

Haga et al. [14] published a study in 1997 in which they advised wait and watch strategy for 1 month in non-syndromic cases as spontaneous reduction of CKD could occur. Conservative management in similar cases was advocated by Bhatia et al. [15], while Cheng and Ko [8] and Bhatti et al. [16] in 2018 have proposed initiating the reduction treatment as early as 


\section{Case Reports in Orthopedic Research}

\begin{tabular}{l|l}
\hline Case Rep Orthop Res 2021;4:138-144 \\
\hline DOI: 10.1159/000516134 & $\begin{array}{l}\text { (c) 2021 The Author(s). Published by S. Karger AG, Basel } \\
\text { www.karger.com/cio }\end{array}$ \\
\hline
\end{tabular}

Tiwari et al.: Successful Early Non-Operative Management of Congenital Knee Dislocation

$24 \mathrm{~h}$ after birth. We feel that like in ponseti casting for clubfoot, we can wait for at least a week to allow the delicate skin of newborn to mature enough to be able to tolerate casting.

Treatment of children with CKD is started early with conservative methods including serial manipulation and casting. In our opinion, surgical procedures should be reserved for resistant cases, especially in older age.

\section{Conclusion}

We have been able to demonstrate that if initiated early, within the first few weeks of life, the congenital deformity of the knee can be managed conservatively with serial casting even in the higher grades of classification of CKD. However, one has to remain vigilant about the possible complications. In non-responsive cases with higher grades, surgical intervention like $\mathrm{V}$-Y quadricepsplasty and percutaneous quadriceps recession may be required.

\section{Statement of Ethics}

The patient's parents have provided written informed consent for publication of this case report and any accompanying images.

\section{Conflict of Interest Statement}

The authors have no conflict of interest to declare.

\section{Funding Sources}

The authors received no financial support for the research and/or authorship of this article.

\section{Author Contributions}

Punit Tiwari: conceptualization, orthopaedic management, methodology, literature review, project administration, resources, and writing - original draft. Harmeet Kaur: conceptualization, radiology investigations, literature review, preparing the figures, and writing original draft. Sergey S. Leonchuk: supervision, formal analysis and validation, literature review, review \& editing the manuscript. All authors discussed, provided critical feedback, contributed and helped shape the final manuscript.

\section{References}

1 Abdelaziz TH, Samir S. Congenital dislocation of the knee: a protocol formanagement based on degree of knee flexion. J Child Orthop. 2011;5:143-9.

2 Curtis BH, Fisher RL. Heritable congenital tibiofemoral subluxation. Clinicalfeatures and surgical treatment. J Bone Joint Surg. 1970;52-A:1104-14.

3 Kaissi AA, Ganger R, Klaushofer K, Grill F. The management of knee dislocation in a child with Larsen syndrome. Clinics. 2011;66(7):1295-9.

4 Shah NR, Limpaphayom N, Dobbs MB. A minimally invasive treatment protocol for the congenital dislocation of the knee. J Pediatr Orthop. 2009;29(7):720-5.

\section{Karger's}


5 Oetgen ME, Walick KS, Tulchin K, Karol LA, Johnston CE. Functional results after surgical treatment for congenital knee dislocation. J Pediatr Orthop. 2010;30(3):216-23.

6 Drennan JC. Congenital dislocation of the knee and patella. Instr Course Lect. 1993;42:517-24.

7 Katz MP, Grogono BJ, Soper KC. The etiology and treatment of congenital dislocation of the knee. J Bone Joint Surg Br. 1967 Feb;49(1):112-20.

8 Cheng CC, Ko JY. Early reduction of congenital dislocation of the knee within 24 hours of birth. Chang Gung Med J. 2010 May-Jun;33(3):266-73.

9 Madadi F, Tahririan MA, Karami M, Madadi F. Complicated congenital dislocation of the knee: a case report. Arch Bone Jt Surg. 2016;4(4):396-8.

10 Mehrafshan M, Wicart P, Ramanoudjame M, Seringe R, Glorion C, Rampal V. Congenital dislocation of the knee at birth: part I: clinical signs and classification. Orthop Traumatol Surg Res. 2016;102(5):631-3.

11 Rumiantcev NJ, Kruglov IJ, Omarov GG. Congenital dislocation of the knee: prenatal diagnostics and treatment at an early age. Pediatr Traumatol Orthop Reconstr Surg. 2017;5(2):26-35.

12 Tiwari P, Leonchuk SS, Kaur H, Sharma G. Congenital knee dislocation: which classification to follow? Open Orthop J. 2020;14(1):58-9.

13 Bell MJ, Atkins RM, Sharrard WJ. Irreducible congenital dislocation of the knee. Aetiology and management. J Bone Joint Surg Br. 1987;69(3):403-6.

14 Haga N, Nakamura S, Sakaguchi R, Yanagisako Y, Taniguchi K, Iwaya T. Congenital dislocation of the knee reduced spontaneously or with minimal treatment. J Pediatr Orthop. 1997;17(1):59-62.

15 Bhatia RK, Pyman J, Gargan MF, Witherow PJ. Cases of congenital dislocation of the knee (CDK) not associated with clubfoot, arthrogryposis multiplex congenita, and Larsen's syndrome can be treated conservatively. J Pediatr Orthop. 1998;18(2):273-4.

16 Bhatti A, Maqsood K, Hassan Shaikh M, Ali P, Ali Z, Yousuf Bhatti M. The earliest age limit for manipulation and casting to avoid surgical intervention in idiopathic congenital knee dislocation. J Pak Orthop Assoc. 2018; $30(02): 75-81$. 